\title{
A study on minimum time regulation of a bounded congested road with upstream flow control
}

\author{
Shu-Xia Tang, Alexander Keimer, Paola Goatin and Alexandre M. Bayen
}

\begin{abstract}
This article is motivated by the practical problem of controlling traffic flow by imposing restrictive boundary conditions. For a one-dimensional congested road segment, we study the minimum time control problem of how to control the upstream vehicular flow appropriately to regulate the downstream traffic into a desired (constant) free flow state in minimum time. We consider the Initial-Boundary Value Problem (IBVP) for a scalar nonlinear conservation law, associated to the Lighthill-Whitham-Richards (LWR) Partial Differential Equation (PDE), where the left boundary condition, also treated as a valve for the traffic flow from the upstream, serves as a control. Besides, we set absorbing downstream boundary conditions. We prove first a comparison principle for the solutions of the considered IBVP, subject to comparable initial, left and right boundary data, which provides estimates on the minimal time required to control the system. Then we consider a (sub-) optimal control problem and we give numerical results based on Godunov scheme. The article serves as a starting point for studying time-optimal boundary control of the LWR model and for computing numerical results.
\end{abstract}

Index Terms - Congested road; conservation law; LWR PDE; IBVP; boundary control; minimum time control.

\section{INTRODUCTION}

Macroscopic evolution of vehicular traffic on road networks can be modeled by treating the flow of vehicles as a continuum. Inspired from fluid dynamics, nonlinear conservation laws can be employed to describe the spatiotemporal evolution of macroscopic quantities such as the vehicle density [11], [15], [16].

Traffic management is of great importance to reduce road congestion, which may have a dramatic impact on both economic and societal aspects. Modern control techniques not only rely on the aforementioned sound mathematical modeling but may also benefit from efficient transportation control algorithms. Regarding the specific problem pf driving a congested road segment to a desired free flow profile, a series of open problems exist such as how to route traffic flow, how much traffic to release on a specific road, etc.. From a control perspective, a controller can be located at boundaries or inner segments of the road. While the inner controllers in general act on the vehicle speed limit, the boundary controllers act mainly on the traffic flow routing.

S.-X. Tang is with Inria Sophia Antipolis-Méditerranée, France; Department of Electrical Engineering \& Computer Sciences, University of California, Berkeley, USA; and Department of Mechanical Engineering, Texas Tech University, USA. (shuxiatang. control@gmail.com) . A. Keimer and A. Bayen are with Department of Civil \& Environmental Engineering, University of California, Berkeley, USA. (\{bayen, keimer\}aberkely.edu) . P. Goatin is with Inria Sophia AntipolisMéditerranée, Université Côte d'Azur, Inria, CNRS, LJAD, Sophia Antipolis Cedex, France. (paola.goatin@inria.fr).
Although traffic modeling needs nonlinear PDEs, a large amount of boundary estimation and control results rely on linearized equations, see e.g. [5], [19], which only manage to stabilize the dynamics in the vicinity of an equilibrium point and do not allow to drive the system from a congested phase to a free-flow regime. On the other hand, few authors consider the congestion alleviation as a PDE boundary control problem. Based on an investigation on the attainable set for scalar nonlinear conservation laws with boundary control on the spatial interval $[0,+\infty[$ in [1], Ancona and Marson further considered the asymptotic stabilization of nonlinear systems of conservation laws by controls acting at a single boundary point on the bounded time-varying spatial interval in [2]. Interested readers can find in [8] a discussion on the asymptotic stabilization near a constant state of systems of conservation laws defined on a fixed bounded spatial interval, following a comprehensive analysis on the boundary controllability of entropy weak solutions. Recently, the stabilization of weak entropy solutions to the scalar nonlinear conservation laws subject to two boundary conditions on a bounded spatial interval has been discussed in [6], using the Lyapunov method.

The aim of this article is to find optimal upstream boundary controls to reach the desired free flow condition in the smallest possible time. It is worth noting that the upstream controllers, acting on the inflow, can be realized for example by enforcing traffic lights (the so-called "metering") and traffic signal controls.

\section{PREliminaries}

Let $\rho:=\rho(t, x)$ denote the vehicle density, i.e. the number of vehicles per unit spatial length at time-space coordinate $(t, x) \in] 0, T[\times] 0, L\left[\right.$ for given $T, L \in \mathbb{R}_{>0}$, and let $f(\rho):=$ $\rho v(\rho)$ denote the traffic flow flux, i.e., the number of vehicles passing through the point $x$ per unit time, $v(\rho)$ denoting the mean traffic speed. The conservation of vehicle number leads to the scalar nonlinear conservation law

$$
\partial_{t} \rho(t, x)+\partial_{x} f(\rho(t, x))=0 .
$$

This results in the classical LWR equation, named after Lighthill and Whitham [15] and Richards [16]. The interested readers are referred to [11] for some detailed mathematical theory on the model.

\section{A. IBVP with boundary control}

We impose boundary conditions given by upstream and downstream traffic densities at the endpoints of the roadway in consideration. The left (upstream) boundary condition is 
considered as the control, which corresponds to a routing policy in the transportation field. On the other side, we set absorbing downstream boundary conditions.

Definition II.1 (IBVP for LWR model). Let $L, T \in \mathbb{R}_{>0}$. Consider the following IBVP posed on $\left.\Omega_{T}:=\right] 0, T[\times] 0, L[$ :

$$
\begin{aligned}
\partial_{t} \rho(t, x)+\partial_{x} f(\rho(t, x)) & =0 & & (t, x) \in \Omega_{T}, \\
\rho(0, x) & =\rho_{0}(x) & & x \in] 0, L[, \\
\rho(t, 0) & =u(t) & & t \in] 0, T[, \\
\rho(t, L) & =\rho_{r}(t) & & t \in] 0, T[,
\end{aligned}
$$

where $\rho: \Omega_{T} \rightarrow \mathbb{R}_{\geq 0}$ denotes the traffic state, $\left.\rho_{0}:\right] 0, L[\rightarrow$ $\mathbb{R}_{>0}$ the initial datum, $\left.u:\right] 0, T\left[\rightarrow \mathbb{R}_{>0}\right.$ the LHS boundary datum (i.e. the control), $\left.\rho_{r}:\right] 0, T\left[\rightarrow \mathbb{R}_{>0}\right.$ the RHS boundary datum, and $f: \mathbb{R}_{\geq 0} \rightarrow \mathbb{R}_{\geq 0}$ the flux function.

For the following analysis we assume

Assumption II.1 (Flux, initial and LHS boundary datum). Assume for the scalar IBVP in Definition II.1 that

- $f(\rho):=V \rho\left(1-\frac{\rho}{\rho_{\max }}\right)$ with the free-flow speed $V \in$ $\mathbb{R}_{>0}$ and maximal density $\rho_{\max } \in \mathbb{R}_{>0}$,

- $\rho_{0} \in B V(] 0, L[): 0 \leq \rho_{0}(x) \leq \rho_{\max }$, a.e. $\left.x \in\right] 0, L[$,

- $\rho_{r} \in B V(] 0, T[): 0 \leq \rho_{r}(t) \leq \rho_{c r}$, a.e. $\left.t \in\right] 0, T[$, where $\rho_{c r}:=\frac{\rho_{\max }}{2}$ denotes the critical density as the stationary point of the concave flux function $f$.

Remark II.1 (Generalizations). The above assumptions are considered for simplicity. Most of the results would remain valid for a general concave flux function satisfying $f(0)=$ $f\left(\rho_{\max }\right)=0$.

Definition II.2 (Free and congested flows). Consider a traffic system with the density function $\rho(t, x),(t, x) \in \Omega_{T}$ and flux function as in Assumption II.1. We say the traffic status $\rho$ is in free flow at time $t \in] 0, T[$ iff

$$
\left.\rho(t, x) \leq \rho_{c r} \quad \text { a.e. } x \in\right] 0, L[\text {. }
$$

Otherwise it is in congested flow.

In congested traffic flow the corresponding characteristics move backwards, while in the case of free flow the characteristics move forwards or do not move (at critical density).

We are concerned about the problem of regulating a congested initial traffic state into some desired free flow. Therefore, we make the following assumptions on the initial and boundary data of the IBVP in Definition II.1

Assumption II.2 (Initial and boundary data). Let $L, T \in$ $\mathbb{R}_{>0}$ and consider the IBVP in Definition II.1. In addition to Assumption II.1. we assume that

$$
\begin{array}{clrl}
\rho_{0}(x) & \left.\in] \rho_{c r}, \rho_{\max }\right] & & x \in] 0, L[\text { a.e. } \\
u(t) & \in\left[0, \rho_{c r}\right] & t \in] 0, T[\text { a.e.. }
\end{array}
$$

Moreover, we define the admissible control set as follows.

Definition II.3 (Admissible control set). Under Assumption II.2, the admissible control set is given by $\mathcal{U}:=$ $B V(] 0, T\left[;\left[0, \rho_{c r}\right]\right)$.
The definition of a solution for the IBVP follows from [4].

Definition II.4. Let $L, T>0, \rho_{0} \in B V(] 0, L[)$ and $u, \rho_{r} \in$ $B V(] 0, T[)$. A function $\rho \in B V(] 0, T[\times] 0, L[)$ is a weak entropy solution to the IBVP in Definition II.1 if for every test function $\phi \in C^{1}\left([0, T] \times[0, L] ; \mathbb{R}_{\geq 0}\right), \phi(T, x)=0 \forall x \in$ $[0, L]$ and for every $\kappa \in \mathbb{R}$, it holds that

$$
\begin{aligned}
& \int_{0}^{\tau} \int_{0}^{L}\left(|\rho-\kappa| \partial_{t} \phi+\operatorname{sgn}(\rho-\kappa)(f(\rho)-f(\kappa)) \partial_{x} \phi\right) \mathrm{d} x \mathrm{~d} t \\
& +\int_{0}^{L}\left|\rho_{0}(x)-\kappa\right| \phi(0, x) \mathrm{d} x \\
& +\int_{0}^{\tau} \operatorname{sgn}(u(t)-\kappa)(f(\rho(t, 0+)-f(\kappa)) \phi(t, 0) \mathrm{d} t \\
& -\int_{0}^{\tau} \operatorname{sgn}\left(\rho_{r}(t)-\kappa\right)(f(\rho(t, L-)-f(\kappa)) \phi(t, 0) \mathrm{d} t \geq 0 .
\end{aligned}
$$

A well-posedness theorem is presented in [17] for general IBVP posed on a finite segment. Below we adapt the result to the IBVP under Definition II.4.

Theorem II.1. [17] Let L,T $>0$ and $\rho_{0} \in$ $B V(] 0, L[), u, \rho_{r} \in B V(] 0, T[)$. Then, the IBVP problem in Definition II.1 admits a unique solution $\rho \in$ $B V(] 0, T[\times] 0, L[)$ in the sense of Definition II.4, which depends Lipschitz continuously in the $L^{1}$-norm from initial and boundary data.

Definition II.5 (Notation). The solution for given data $\rho_{0}, u, \rho_{r}$ at $\left.(t, x) \in\right] 0, T[\times] 0, L[$ is denoted by $\rho\left(t, x ; \rho_{0}, u, \rho_{r}\right)$ to indicate the functional dependency of the solution w.r.t. initial datum and boundary data.

\section{B. Godunov Scheme}

The Godunov scheme [12] is a finite volume numerical scheme, based on exact solutions to Riemann problems. Below we describe the procedure for our IBVP. In this article, we use uniform temporal and spatial meshes, noting that more general meshes can be considered.

Let that $\Delta x=L / N$ and $\Delta t=T / M$ for some $N, M \in$ $N_{>0}$. We set

$$
\begin{aligned}
t^{n} & =n \Delta t, & & n \in\{0, \ldots, M\}, \\
x_{j} & =\left(j-\frac{1}{2}\right) \Delta x, & & j \in\{1,2, \cdots, N\}, \\
x_{j-1 / 2} & =j \Delta x, & & j \in\{0,1, \cdots, N\}, \\
\lambda & =\frac{\Delta t}{\Delta x} . & &
\end{aligned}
$$

the space and time mesh points, and

$$
x_{j}:=\left(j+\frac{1}{2}\right) \Delta x, j \in\{1, \cdots, N-1\}
$$

the cell boundaries, so that $x_{\frac{1}{2}}=0, x_{N-\frac{1}{2}}=L$.

In addition, we require the following condition:

Definition II.6 (CFL condition [10]). $\Delta t, \Delta x$ satisfy the Courant-Friedrichs-Lewy (CFL) condition iff

$$
\Delta t \max _{\rho \in[0, R]}\left|f^{\prime}(\rho)\right| \leq \Delta x \Longleftrightarrow V \lambda \leq 1
$$


Definition II.7 (Godunov-type numerical flux). Given the concave flux function $f: \mathbb{R}_{\geq 0} \rightarrow \mathbb{R}$ as in Assumption II.1. the corresponding Godunov numerical flux is defined as:

$$
\mathcal{F}(a, b):=\min \left\{f\left(\min \left\{a, \rho_{c r}\right\}\right), f\left(\max \left\{b, \rho_{c r}\right\}\right)\right\} .
$$

We present a lemma that will be used to prove Theorem V.1, which follows directly from Definition II.7 The proof is omitted here.

Lemma II.1. The numerical flux $\mathcal{F}$ as in Definition II.7 is increasing with respect to the first argument and decreasing with respect to the second.

Definition II.8 (Godunov scheme). Recalling Definitions II.6 and II.7. we define the discretization of the initial datum for $j \in\{1,2, \ldots, N\}$ as

$$
\rho_{j}^{0}:=\frac{1}{\Delta x} \int_{(j-1 / 2) \Delta x}^{(j+1 / 2) \Delta x} \rho_{0}(x) \mathrm{d} x=\frac{1}{\Delta x} \int_{x_{j-1 / 2}}^{x_{j+1 / 2}} \rho_{0}(x) \mathrm{d} x
$$

and the boundary data for $n \in\{0, \ldots, M\}$ as

$$
\begin{aligned}
& \rho_{0}^{n}:=u^{n}=\frac{1}{\Delta t} \int_{t^{n}}^{t^{n+1}} u(t) \mathrm{d} t \\
& \rho_{N+1}^{n}:=\rho_{r}^{n}=\frac{1}{\Delta t} \int_{t^{n}}^{t^{n+1}} \rho_{r}(t) \mathrm{d} t .
\end{aligned}
$$

In addition, introducing for $j \in\{2,3, \ldots, N-1\}$

$$
\begin{aligned}
H_{1}\left(\rho^{n}, u^{n}, \rho_{r}^{n}\right) & :=\frac{1}{\Delta x}\left(\mathcal{F}\left(u^{n}, \rho_{1}^{n}\right)-\mathcal{F}\left(\rho_{1}^{n}, \rho_{2}^{n}\right)\right), \\
H_{j}\left(\rho^{n}, u^{n}, \rho_{r}^{n}\right) & :=\frac{1}{\Delta x}\left(\mathcal{F}\left(\rho_{j-1}^{n}, \rho_{j}^{n}\right)-\mathcal{F}\left(\rho_{j}^{n}, \rho_{j+1}^{n}\right)\right), \\
H_{N}\left(\rho^{n}, u^{n}, \rho_{r}^{n}\right) & :=\frac{1}{\Delta x}\left(\mathcal{F}\left(\rho_{N-1}^{n}, \rho_{N}^{n}\right)-\mathcal{F}\left(\rho_{N}^{n}, \rho_{r}^{n}\right)\right),
\end{aligned}
$$

the Godunov scheme is defined by

$$
\rho_{j}^{n+1}=\rho_{j}^{n}+\Delta t H_{j}\left(\rho^{n}, u^{n}, \rho_{r}^{n}\right),
$$

for $(n, j) \in\{0, \ldots, M-1\} \times\{1, \ldots, N\}$. The approximate solution is given by

$$
\rho_{\Delta}(t, x):=\sum_{n=0}^{M-1} \sum_{j=1}^{N} \rho_{j}^{n} \chi_{\left[t^{n}, t^{n+1}[\right.}(t) \chi_{\left[x_{j-1 / 2}, x_{j+1 / 2}[\right.}(x)
$$

for $(t, x) \in \Omega_{T}$.

\section{Explicit Solution to the IBVP with Constant INITIAL DATUM AND PIECEWISE CONSTANT CONTROL}

We are interested in regulating the possibly congested traffic system to a desired free flow profile, by means of the upstream boundary control. From now on, for simplicity, we set $\rho_{\mathrm{r}}(t)=0, \forall t \in[0, T]$ and $v(\rho)=1-\rho$, unless otherwise indicated.

In this section, we consider a sample problem, which is to drive the congested initial condition $\left.\left.\rho_{0} \in\right] \rho_{c r}, \rho_{\max }\right]$ to some constant free flow state $\rho_{1} \in\left[0, \rho_{c r}[\right.$, using boundary controls $u=u(t) \in\left\{0, \rho_{1}\right\}$. We will consider the following control choices:
1) $u(t)=\rho_{1}, \forall t \in[0, T]$.

Then, the corresponding Riemann problem at $x=0$ is

$$
\begin{gathered}
\left.\partial_{t} \rho(t, x)+\partial_{x} f(\rho(t, x))=0, \quad(t, x) \in\right] 0, \infty[\times \mathbb{R} \\
\rho(0, x)=\left\{\begin{array}{ll}
\rho_{1} & \text { if } x<0, \\
\rho_{0} & \text { if } x>0,
\end{array} \quad x \in \mathbb{R},\right.
\end{gathered}
$$

and the Riemann problem at $x=L$ is

$$
\begin{gathered}
\left.\partial_{t} \rho(t, x)+\partial_{x} f(\rho(t, x))=0, \quad(t, x) \in\right] 0, \infty[\times \mathbb{R} \\
\rho(0, x)= \begin{cases}\rho_{0} & \text { if } x<L, \\
0 & \text { if } x>L,\end{cases}
\end{gathered}
$$

Recall that $0<\rho_{1}<\rho_{0}$ : the solution to III.1) is given by

$$
\rho(t, x)= \begin{cases}\rho_{1} & \text { if } x<\left(1-\rho_{0}-\rho_{1}\right) t \\ \rho_{0} & \text { if } x>\left(1-\rho_{0}-\rho_{1}\right) t\end{cases}
$$

which contains a shock wave. The solution to (III.2) is

$$
\rho(t, x)= \begin{cases}\rho_{0} & \text { if } 0<x<\left(1-2 \rho_{0}\right) t+L, \\ \frac{t-x+L}{2 t} & \text { if }\left(1-2 \rho_{0}\right) t+L<x<t+L, \\ 0 & \text { if } x>t+L,\end{cases}
$$

generating a rarefaction wave. Depending on the choice of the boundary control value $\rho_{1}$, we distinguish two cases:

a) If $\rho_{1} \leq 1-\rho_{0}$, the shock intersects with the right-side rarefaction wave at $\frac{L}{\rho_{0}-\rho_{1}}$, and thus for $0<t<\frac{L}{\rho_{0}-\rho_{1}}$,

$\rho(t, x)= \begin{cases}\rho_{1} & \text { if } 0<x<\left(1-\rho_{0}-\rho_{1}\right) t, \\ \rho_{0} & \text { if }\left(1-\rho_{0}-\rho_{1}\right) t<x<\left(1-2 \rho_{0}\right) t+L, \\ \frac{t-x+L}{2 t} & \text { if }\left(1-2 \rho_{0}\right) t+L<x<L .\end{cases}$

For $t>\frac{L}{\rho_{0}-\rho_{1}}$, it can be derived that

$\rho(t, x)=\left\{\begin{array}{l}\rho_{1} \\ \text { if } 0<x<\left(1-2 \rho_{1}\right) t-2 \sqrt{L\left(\rho_{0}-\rho_{1}\right) t}+L, \\ \frac{t-x+L}{2 t}, \\ \text { if }\left(1-2 \rho_{1}\right) t-2 \sqrt{L\left(\rho_{0}-\rho_{1}\right) t}+L<x<L .\end{array}\right.$

Note that the critical case when $\rho_{1}=1-\rho_{0}$ can be considered as a degenerate one. The settling time is $T_{1}=$ $L \frac{4\left(\rho_{0}-\rho_{1}\right)}{\left(1-2 \rho_{1}\right)^{2}}$.

b) If $\rho_{1}>1-\rho_{0}$, the controller, even if activated at $t=0$, takes effect at the critical time $T_{2,1}=\frac{L}{1-2 \rho_{1}}$, when the rightside rarefaction wave with $\rho(t, x)=\frac{t-x+L}{2 t}=1-\rho_{1}$ (i.e. the line $\left.x=\left(2 \rho_{1}-1\right) t+L\right)$ meets the axis $x=0$. For $t>T_{2,1}$, the solution is

$\rho(t, x)=\left\{\begin{array}{l}\rho_{1}, \\ \text { if } 0<x<\left(1-2 \rho_{1}\right) t-2 \sqrt{L\left(1-2 \rho_{1}\right) t}+L, \\ \frac{t-x+L}{2 t}, \\ \text { if }\left(1-2 \rho_{1}\right) t-2 \sqrt{L\left(1-2 \rho_{1}\right) t}+L<x<L .\end{array}\right.$

The settling time is $T_{2}=\frac{4 L}{1-2 \rho_{1}}$.

2) Return Method. An alternative approach is to first evacuate the roadway entirely, i.e., setting the left side inflow 
to be $u(t)=0$ for $t \in[0, \bar{t}]$ for some $\bar{t}>0$; and then to allow appropriate traffic $u(t)=\rho_{1}$, for $t>\bar{t}$, into the roadway, which will drive the flow density profile into the desired one. This is referred to as the return method [9, Chapter 6].

a) First, we calculate the time when all vehicles exit the roadway under consideration. The unique weak entropy solution to the IBVP with $u(t)=0, \forall t \geq 0$, is given by

$$
\rho(t, x)= \begin{cases}0 & \text { if } 0<x<\left(1-\rho_{0}\right) t, \\ \rho_{0} & \text { if }\left(1-\rho_{0}\right) t<x<\left(1-2 \rho_{0}\right) t+L, \\ \frac{t-x+L}{2 t} & \text { if }\left(1-2 \rho_{0}\right) t+L<x<L,\end{cases}
$$

for $0<t<\frac{L}{\rho_{0}}$ and

$$
\rho(t, x)= \begin{cases}0 & \text { if } 0<x<t-2 \sqrt{L \rho_{0} t}+L, \\ \frac{t-x+L}{2 t} & \text { if } t-2 \sqrt{L \rho_{0} t}+L<x<L,\end{cases}
$$

for $t>\frac{L}{\rho_{0}}$. Straightforward calculation tells that all the vehicles have left the road $[0, L]$ after $\bar{t}=T_{3,1}=4 L \rho_{0}$.

b) Immediately after all vehicles exit the roadway at time $T_{3,1}$, we apply the constant boundary controller $u(t)=\rho_{1}$, $t>T_{3,1}$. The solution exhibits a rarefaction wave as follows:

$\rho(t, x)= \begin{cases}\rho_{1} & \text { if } 0<x<\left(1-2 \rho_{1}\right)\left(t-T_{3,1}\right), \\ \frac{t-T_{3,1}-x}{2\left(t-T_{3,1}\right)} & \text { if }\left(1-2 \rho_{1}\right)\left(t-T_{3,1}\right)<x<t-T_{3,1}, \\ 0 & \text { if } x>t-T_{3,1} .\end{cases}$

Note that the vehicle density reaches the uniform value $\rho_{1}$ after $T_{3}=T_{3,1}+\frac{L}{1-2 \rho_{1}}=L\left(4 \rho_{0}+\frac{1}{1-2 \rho_{1}}\right)$.

3) Optimized Return Method. For sake of efficiency, we propose to apply the controller with constant value $\rho_{1}$ earlier than $T_{3,1}$.

Indeed, if we apply the left boundary controller starting from the time $T_{4,1}=L\left(4 \rho_{0}-1\right)$, the incoming traffic does not interact with the vehicles on the road at $t=0$, see Fig. 1 for the illustration. Some straightforward calculations tell that the settling time in this case is $T_{4}=T_{4,1}+\frac{L}{1-2 \rho_{1}}=$ $2 L\left(2 \rho_{0}+\frac{\rho_{1}}{1-2 \rho_{1}}\right)$. Note that the settling time $T_{4}$ decreases if the initial data $\rho_{0}$ is smaller or if the control $\rho_{1}$ is smaller since $\rho_{1}$ also denotes the real-time car density that we allow to enter from the left boundary.

We can now compare the settling times $T_{i}, i=1, \ldots, 4$, computed above. It can immediately be derived that $T_{3}>$ $T_{4}$. For the case of $\rho_{1}<1-\rho_{0}$, we have $T_{4}<T_{1}$ and $T_{3} \geq T_{1}$ if $16 \rho_{0} \rho_{1}-16 \rho_{0} \rho_{1}^{2}-2 \rho_{1} \leq 1$ or $T_{3}<T_{1}$ if $16 \rho_{0} \rho_{1}-16 \rho_{0} \rho_{1}^{2}-2 \rho_{1}>1$. For the case of $\rho_{1}>1-\rho_{0}$, we derive that $T_{4}<T_{2}$ and $T_{3} \geq T_{2}$ if $4 \rho_{0}\left(1-2 \rho_{1}\right) \geq 3$ or $T_{3}<T_{2}$ if $4 \rho_{0}\left(1-2 \rho_{1}\right)<3$. Therefore, the control given by the Optimized Return Method drives the system into the free flow $\rho_{1}$ earlier than the other studied options.

\section{Problem Statement}

Consider a given target uniform free flow profile $\rho_{1} \in$ $\left[0, \rho_{c r}[\right.$. Given a control function $u \in \mathcal{U}$, denote by $T^{\min }\left(u ; \rho_{0}\right)$ the minimum/first time (w.r.t. $u$ ) to reach the target flow as

$$
T^{\min }\left(u ; \rho_{0}\right)=\inf _{t>0}\left\{\rho\left(t, x ; u, \rho_{0}\right)=\rho_{1}, x \in\right] 0, L[\},
$$

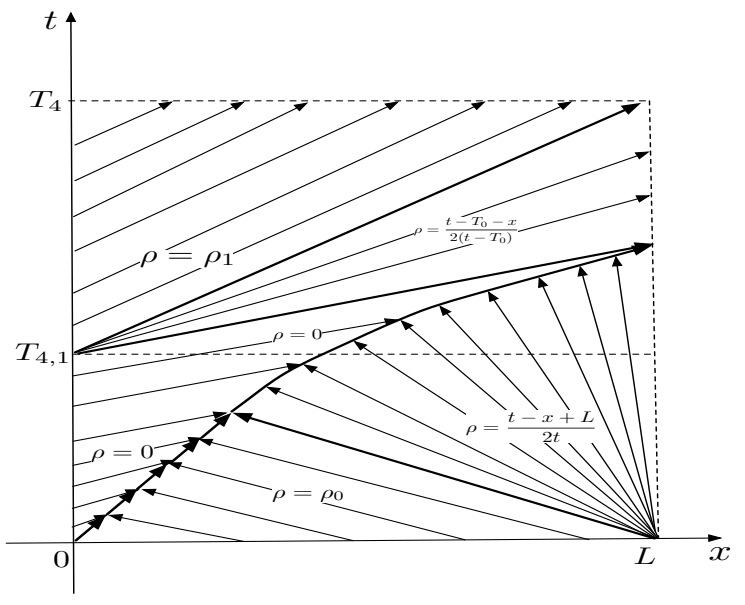

Fig. 1. Solution to the IBVP in Definition II.1 by applying the left boundary controller following the optimized return method.

where $\rho\left(t, x ; u, \rho_{0}\right):=\rho\left(t, x ; u, \rho_{0}, 0\right)$ denotes the weak entropy solution of IBVP corresponding to the initial datum $\rho_{0}$, upstream boundary control $u$ and downstream boundary condition $\rho_{\mathrm{r}}(t) \equiv 0$. From Section III, we can conclude that, for any $\rho_{0} \in B V(] 0, L\left[;\left[0, \rho_{\max }\right]\right)$, there exists a control $u \in \mathcal{U}$ such that $T^{\min }\left(u ; \rho_{0}\right)<\infty$.

We aim at finding the minimum time required to drive the congested road to the desired free flow state and as well the corresponding admissible boundary control that can achieve this goal. The control leading to the minimal time is called minimum time control and will be denoted as $u^{\text {opt }}$.

Considering the possible spill-back phenomenon, where the boundary control cannot be effective in some time interval, we can always find another $u^{\prime} \in \mathcal{U}$, taking the same value as $u$ in all other time interval except for this one, achieving the same minimum time for the desired free flow profile. As a result, if such control exists, it is not necessarily unique.

\section{Estimate of the Minimum Time}

In this section, we give a rough upper bound of the minimum time.

\section{A. A comparison principle}

First, we prove a comparison principle for entropyadmissible solutions $\rho(t, x)$ to the IBVP. (For an alternative proof, see [13, Theorem 4.1].)

Theorem V.1 (Comparison Principle). For initial data $\rho_{0}, \rho_{0}^{\prime} \in B V(] 0, L[)$ and boundary data $u, u^{\prime}, \rho_{r}, \rho_{r}^{\prime} \in$ $B V(] 0, T[)$, such that

$$
\begin{aligned}
\rho_{0}(x) & \leq \rho_{0}^{\prime}(x) & & \text { a.e. } x \in] 0, L[ \\
u(t) & \leq u^{\prime}(t), \rho_{r}(t) \leq \rho_{r}^{\prime} & & \text { a.e. } t \in] 0, T[,
\end{aligned}
$$

it holds

$$
\rho\left(t, x ; \rho_{0}, u, \rho_{r}\right) \leq \rho\left(t, x ; \rho_{0}^{\prime}, u^{\prime}, \rho_{r}^{\prime}\right) \quad \text { a.e. }(t, x) \in \Omega_{T} .
$$

Proof. The approximate solutions to the IBVP in Definition II.1 are constructed by Godunov method, detailed in 
Subsection II-B For $n=0$, let $\rho_{j}^{0}, \rho_{j}^{0 \prime}, j \in\{1,2, \ldots, N\}$ be the approximations of the initial data $\rho_{0}, \rho_{0}^{\prime}$ respectively, defined as in Eq. (II.7) Similarly, piece-wise constant (left and right) approximate boundary conditions computed using Eq. (II.8) and Eq. (II.9) will be denoted $u^{n}, u^{n \prime}, \rho_{r}^{n}, \rho_{r}^{n \prime}, n=$ $0, \ldots, M$. Note that the ordering is maintained in the corresponding approximations. More precisely, we have

$$
\begin{array}{ll}
\rho_{j}^{0} \leq \rho_{j}^{0 \prime} & j \in\{1,2, \ldots, N\} \\
u^{n} \leq u^{n \prime} & n \in\{0, \ldots, M\}, \\
\rho_{r}^{n} \leq \rho_{r}^{n \prime} & n \in\{0, \ldots, M\} .
\end{array}
$$

We observe now that applying the scheme in Eq. (II.13) with Eq. (II.10) Eq. (II.12) is equivalent to solving exactly the Cauchy problem

$$
\begin{array}{cl}
\partial_{t} \rho(t, x)=-\partial_{x} f(\rho(t, x)) & (t, x) \in] t^{n}, t^{n+1}[\times \mathbb{R}, \\
\rho\left(t^{n}, x\right)=\rho_{j}^{n} & x \in\left[x_{j-1 / 2}, x_{j+1 / 2}[,\right. \\
& j \in\{0, \ldots, N\}
\end{array}
$$

(setting, with slight abuse of notation, $] x_{-1 / 2}, x_{1 / 2}[:=]-$ $\infty, 0\left[\right.$ and $\left[x_{N+1 / 2}, x_{N+3 / 2}[:=[L,+\infty[)\right.$ and then averaging the solutions on each cell, which gives

$$
\hat{\rho}_{j}^{n+1}=\frac{1}{\Delta x} \int_{x_{j-1 / 2}}^{x_{j+1 / 2}} \rho_{\Delta x}^{n}(x) d x, \quad j=1, \ldots, N .
$$

By the classical comparison principle for Eq. (V.4), see [14], [7], we have that $\rho_{\Delta}(t, x) \leq \rho_{\Delta}^{\prime}(t, x)$ a.e. in ]$t^{n}, t^{n+1}[\times] 0, L\left[\right.$ if $\rho_{\Delta}\left(t^{n}, x\right) \leq \rho_{\Delta x}^{\prime}\left(t^{n}, x\right)$ for a.e. $x \in$ ] $0, L[$. Reasoning by induction, we can conclude that

$$
\left.\rho_{\Delta}(t, x) \leq \rho_{\Delta}^{\prime}(t, x) \quad \text { a.e. in }\right] 0, T[\times] 0, L[.
$$

A direct application of [18, Theorem 5] ensures that $\rho_{\Delta}, \rho_{\Delta}^{\prime}$ converge respectively to the weak entropy solutions $\rho, \rho^{\prime}$ of the corresponding IBVP in $L_{\text {loc }}^{1}([0, T[\times] 0, L[;[0, R])$, as $\Delta x$ goes to zero. By pointwise a.e. convergence, from Eq. (V.5) we conclude that $\rho^{1}(t, x) \leq \rho^{2}(t, x)$ a.e. in $[0, T[\times] 0, L[$.

This comparison principle allows to derive an upper bound of the minimum time.

\section{B. Upper bound on the minimal time}

Given a constant target uniform free flow profile $\rho_{1} \in$ $\left[0, \rho_{c r}[\right.$, let us assume that the initial condition satisfies $\left.\rho_{0}(x) \leq \rho_{M}, \forall x \in\right] 0, L\left[\right.$, for some constant $\rho_{M} \in$ ]$\left.\rho_{c r}, \rho_{\max }\right]$. Let $u_{M}(t)$ be an optimal control in the sense that it regulates the traffic system into the desired free flow no later than any other control within the admissible control set $\mathcal{U}$, that is, for any other control $\bar{u}_{M}(t) \in \mathcal{U}$ applied at the left boundary, if there exists a time $T$ such that the solution $\rho\left(t, x ; \rho_{M, 0}, \bar{u}_{M}\right)=\rho_{1}, \forall t \geq T$, then $\rho\left(t, x ; \rho_{M}, u_{M}\right)=$ $\rho_{1}, \forall t \geq T$. From the comparison principle in Theorem V.1. we get

$$
\rho\left(t, x ; \rho_{0}, u_{M}\right) \leq \rho\left(t, x ; \rho_{M}, u_{M}\right)=\rho_{1},
$$

$\forall t \geq T^{\min }\left(u_{M} ; \rho_{M}\right)$, see IV.1. As seen in Section III if $v(\rho)=1-\rho$, we have

$$
T^{\min }\left(u_{M} ; \rho_{M}\right) \leq 2 L\left(2 \rho_{M}+\frac{\rho_{1}}{1-2 \rho_{1}}\right) .
$$

This means that the minimum time $T^{\min }\left(u_{M} ; \rho_{0}\right)$ to drive the system in Definition II.1 with the initial condition $\rho_{0}(x)$ to the same free flow $\rho_{1}$, using the same control $u_{M}(t)$, satisfies

$$
T^{\min }\left(u_{M} ; \rho_{0}\right) \leq T^{\min }\left(u_{M} ; \rho_{M}\right)
$$

It can then be immediately derived that

$$
T^{\min }\left(u^{\mathrm{opt}} ; \rho_{0}\right) \leq T^{\min }\left(u_{M} ; \rho_{M}\right) \leq 2 L\left(2 \rho_{M}+\frac{\rho_{1}}{1-2 \rho_{1}}\right),
$$

recalling that $u^{\mathrm{opt}}$ denotes the minimum time control.

Furthermore, by replacing the above constant bound $\rho_{M}$ of the initial datum with a closer piece-wise constant initial datum, $\left.\rho_{0}(x) \leq \rho_{M}(x), \forall x \in\right] 0, L\left[\right.$ with $\left.\left.\rho_{M}(x) \in\right] \rho_{c r}, R\right]$, one could derive a closer estimate of the minimal time.

\section{Minimum Time Control}

With the initial condition $\rho_{0} \in B V(] 0, L[;[0, R])$, we compute an optimal control $u^{\mathrm{opt}}(t)$ to regulate the traffic system in Definition II.1 into the desired free flow no later than any other control in the admissible control set. The problem is then posed as the following constrained optimization problem:

$$
u^{\text {opt }} \in \arg \min _{u \in \mathcal{U}}\left\{t \geq 0 ; \rho\left(t, x ; u, \rho_{0}\right)=\rho_{1}, \forall x \in\right] 0, L[\} .
$$

For any control $u \in \mathcal{U}$, the function $\rho\left(t, x ; u, \rho_{0}\right)$ in Eq. (VI.1) is derived by solving the IBVP in Definition II.1. following the Godunov scheme detailed in Subsection II-B.

Instead of solving Eq. (VI.1) directly, which is computationally not affordable, we propose to consider for an arbitrary yet reasonably large enough final time $\left.t_{\mathrm{f}} \in\right] 0, T[$ the following reformulation of the problem:

$$
\min _{u \in \mathcal{U}}\left\|\rho\left(\cdot, * ; u, \rho_{0}\right)-\rho_{1}\right\|_{L^{2}(] 0, t_{\mathrm{f}}[\times] 0, L[)}^{2}+C\|u\|_{L^{2}(] 0, t_{\mathrm{f}}[)}^{2},
$$

where $C \in \mathbb{R}_{>0}$ is a weighting coefficient. Note that the cost function, i.e. the $L^{2}$ norm of the error between the solution and the desired free flow density profile, as the cost function, is penalized by the weighted 2-norm of the boundary control. This ensures sufficient regularity of the control function $u$. If the cost function value is small enough, we consider the returned in-flow control function in the (sub-)optimal sense. Following the comparison principle in $\mathrm{V}-\mathrm{A}$, one can choose $t_{f}=2 L\left(2 \rho_{M}+\frac{\rho_{1}}{1-2 \rho_{1}}\right)$ which is the rough upper bound on the minimal time derived in $\mathrm{V}-\mathrm{B}$.

The next steps are merely involved with tuning the value of $t_{\mathrm{f}}$ (to be smaller) and solving Eq. (VI.2) to find the minimum time and its associated minimum time control. The criterion is to make sure that within any other shorter time interval, the system cannot be regulated any more into the desired free flow profile subject to the preset tolerable error. 
We illustrate these results numerically. We set $L=1$, $N=25$, the CFL to 0.99 and $v(\rho)=1-\rho$. The initial traffic density is taken to be $\left.\rho_{0}(x)=0.7, \forall x \in\right] 0, L[$ and the target uniform free flow density as $\rho_{1}=0.45$.

We solve numerically the problem (VI.1). The initial guess of optimal boundary control is set to be uniformly $u(t)=$ $0.45, \forall t \geq 0$, acknowledging that the MATLAB package fmincon implemented with the spq algorithm might only provide a local optimum.

See Fig. 2 and Fig. 3 for the simulated (sub-)optimal minimum time boundary control by solving Eq. (VI.2) and a 2D $(t, x)$-plot of the density, respectively, where the minimum time is smaller than the one associated with the optimized return method, $T_{4}=2 L\left(2 \rho_{M}+\frac{\rho_{1}}{1-2 \rho_{1}}\right)=9.28$.

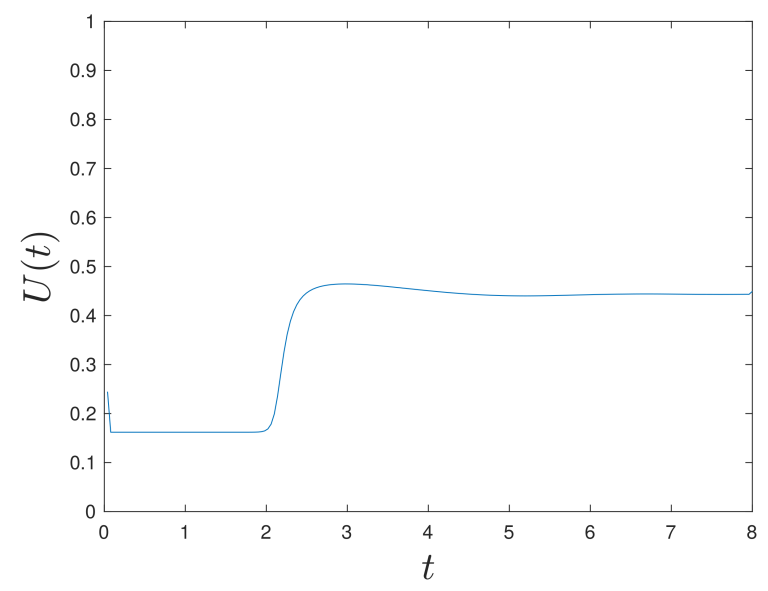

Fig. 2. The simulated (sub-)optimal minimum time boundary control

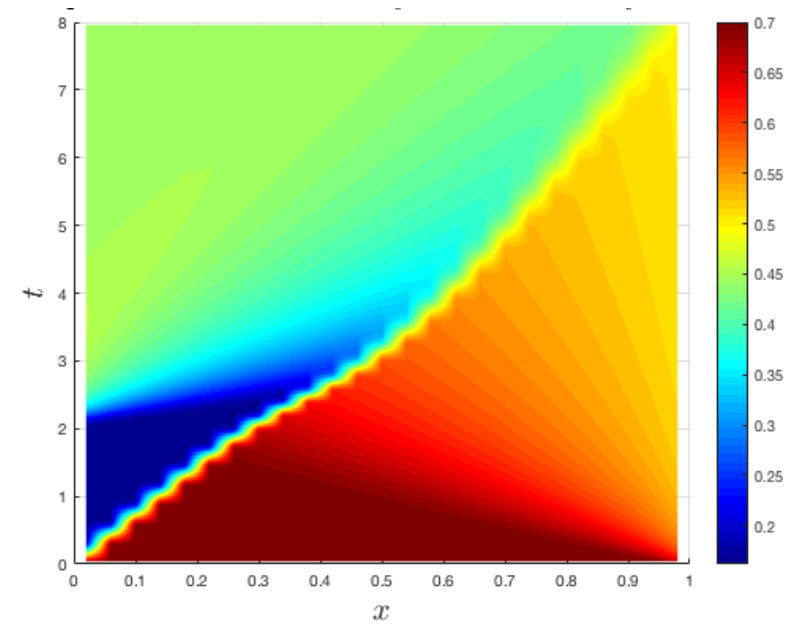

Fig. 3. Plot of the traffic density evolution under minimum time control

\section{CONCLUSION AND FUtURE WORK}

In this article, we present a preliminary study on minimum time regulation of a bounded congested road using upstream flow control. For a given constant free flow profile, a framework is provided to numerically find the (sub-) optimal pair of minimum time and minimum time control. This framework can be extended to any non-constant free flow profile. Motivated by the trade-off between decreasing travel time and increasing traffic load on the road, an ongoing extension is to minimize the control time while maximizing the upstream incoming flow.

Another ongoing investigation and possible extension is to solve directly the minimum time problem (VI.1) through the corresponding Hamilton-Jacobi-Bellman (HJB) equation obtained from the Dynamic Programming Principle (DPP) [3, Chapter 1, Proposition 2.1]: for all $t \in\left[0, T^{\min }\left(\rho_{0}\right)\right]$,

$$
\begin{gathered}
T^{\min }\left(\rho_{0}\right):=\inf _{u \in \mathcal{U}}\left\{t+T^{\min }\left(\rho\left(t, \cdot ; u, \rho_{0}\right)\right)\right\} . \\
\text { REFERENCES }
\end{gathered}
$$

\section{REFERENCES}

[1] F. Ancona and A. Marson. On the attainable set for scalar nonlinear conservation laws with boundary control. SIAM Journal on Control and Optimization, 36(1):290-312, 1998.

[2] F. Ancona and A. Marson. Asymptotic stabilization of systems of conservation laws by controls acting at a single boundary point. Contemporary Mathematics, 426:1, 2007.

[3] M. Bardi and I. Capuzzo-Dolcetta. Optimal control and viscosity solutions of Hamilton-Jacobi-Bellman equations. Springer Science \& Business Media, 2008.

[4] C. Bardos, A.-Y. Leroux, and J.-C. Nédélec. First order quasilinear equations with boundary conditions. Communications in Partial Differential Equations, 4(9):1017-1034, 1979.

[5] F. Belletti, M. Huo, X. Litrico, and A. M. Bayen. Characterization of the convective instability of the Aw-Rascle-Zhang model via spectral analysis. In 2016 American Control Conference (ACC), pages 61276132. IEEE, 2016.

[6] S. Blandin, X. Litrico, M. L. Delle Monache, B. Piccoli, and A. Bayen. Regularity and Lyapunov stabilization of weak entropy solutions to scalar conservation laws. IEEE Transactions on Automatic Control, 62(4):1620-1635, 2017.

[7] A. Bressan. Hyperbolic systems of conservation laws: the onedimensional Cauchy problem, volume 20. Oxford University Press on Demand, 2000.

[8] A. Bressan and G. M. Coclite. On the boundary control of systems of conservation laws. SIAM J. Control Optim., 41(2):607-622, 2002.

[9] J.-M. Coron. Control and nonlinearity. American Mathematical Soc. 2007.

[10] R. Courant, K. Friedrichs, and H. Lewy. Über die partiellen Differenzengleichungen der mathematischen Physik. Math. Ann., 100(1):3274, 1928.

[11] M. Garavello and B. Piccoli. Traffic flow on networks, volume 1. American institute of mathematical sciences Springfield, 2006.

[12] S. K. Godunov. A difference method for numerical calculation of discontinuous solutions of the equations of hydrodynamics. Mat. Sb. (N.S.), 47 (89):271-306, 1959.

[13] C. Imbert and J. Vovelle. A kinetic formulation for multidimensional scalar conservation laws with boundary conditions and applications. SIAM J. Math. Anal., 36(1):214-232, 2004.

[14] S. N. Kružkov. First order quasilinear equations in several independent variables. Mathematics of the USSR-Sbornik, 10(2):217, 1970.

[15] M. J. Lighthill and G. B. Whitham. On kinematic waves. II. A theory of traffic flow on long crowded roads. In Proceedings of the Royal Society of London A: Mathematical, Physical and Engineering Sciences, volume 229, pages 317-345. The Royal Society, 1955.

[16] P. I. Richards. Shock waves on the highway. Operations Res., 4:42-51, 1956.

[17] E. Rossi. Well-posedness of general 1D initial boundary value problems for scalar balance laws. Discrete Contin. Dyn. Syst., 39(6):3577$3608,2019$.

[18] J. Vovelle. Convergence of finite volume monotone schemes for scalar conservation laws on bounded domains. Numerische Mathematik, 90(3):563-596, 2002.

[19] H. Yu, M. Diagne, L. Zhang, and M. Krstic. Bilateral boundary control of moving shockwave in LWR model of congested traffic. arXiv preprint arXiv:1904.04303, 2019. 